
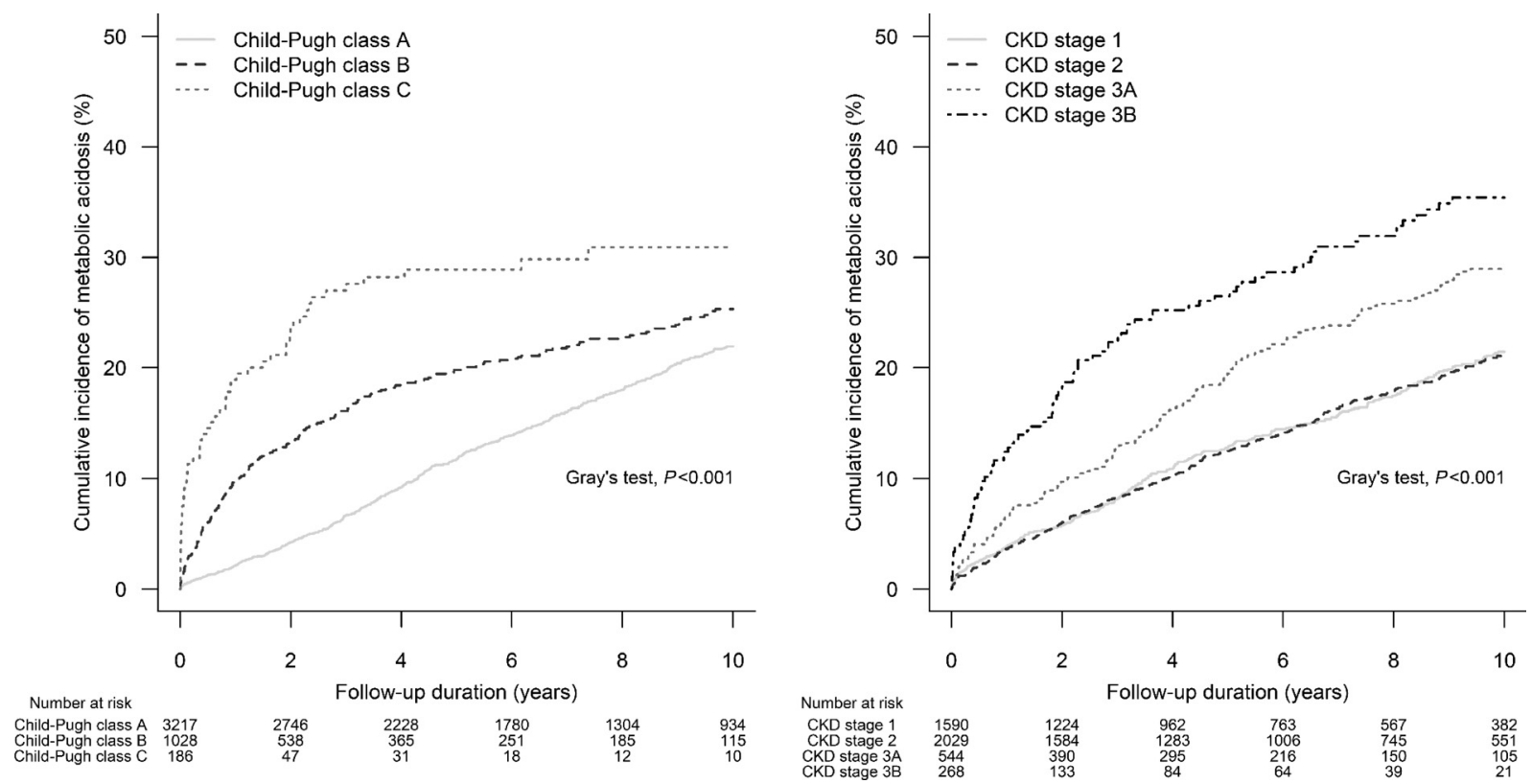

Abstract IDDF2019-ABS-0199 Figure 1 Cirrhosis with different Child-Pugh class and estimated glomerular filtration rate eGFR cate

years; 1,590 (35.9\%), $2,029(45.8 \%), 544(12.3 \%)$ and 268 $(6.0 \%)$ had baseline eGFR $\geq 90,60-89,45-59$ and $30-44$ $\mathrm{mL} / \mathrm{min} / 1.73 \mathrm{~m}^{2} ; 3,217 \quad(72.6 \%), 1,028 \quad(23.2 \%)$ and 186 (4.2\%) were in Child-Pugh class A, B and C at baseline (figure 1). At a median (interquartile range) follow-up of 5.3 (2.0-9.7) years, 1,060 (23.9\%) patients developed metabolic acidosis. In Child-Pugh class $\mathrm{A}$, the risk of metabolic acidosis elevated in eGFR $<45 \mathrm{~mL} / \mathrm{min} / 1.73 \mathrm{~m}^{2}$ (adjusted subdistribution hazard ratio [SHR] 4.02, 95\% CI 2.42-6.68, $\mathrm{P}<0.001$ ). The risk of metabolic acidosis increased in Child-Pugh class $\mathrm{B}$ and $\mathrm{C}$ at any eGFR levels (adjusted SHR ranged from 4.24 to 91.66), particularly in Child-Pugh class $C$ with eGFR $<30$ $\mathrm{mL} / \mathrm{min} / 1.73 \mathrm{~m}^{2}$ (adjusted SHR 91.66, 95\% CI 60.69-138.44, $\mathrm{P}<0.001)$.

Conclusions The risk of metabolic acidosis increases with renal and liver impairment in diabetic patients with CHB-related cirrhosis.

\section{IDDF2019-ABS-0207 FIRST REPORT OF PRIMARY TENOFOVIR RESISTANCE IN A HEPATITIS B VIRAL HEPATITIS PATIENT FROM INDIA WITHOUT HUMAN IMMUNODEFICIENCY VIRUS CO- INFECTION}

${ }^{1}$ Richa Sharma*, ${ }^{2}$ Vijay Sharma, ${ }^{3}$ Gaurav Gandhi. ${ }^{1}$ Public health foundation of India, Jaipur, Rajasthan, India; ${ }^{2}$ Regional Institute of Health Medicine and Research, Jaipur, Rajasthan, India; ${ }^{3}$ Medipulse hospital, Jodhpur, Rajasthan, India

\subsection{6/gutjnl-2019-IDDFabstracts.282}

Background Tenofovir confers potent and durable HBV-DNA suppression; Levels of Tenofovir resistance in individuals with viral failure ranged from $20 \%$ in Europe to more than $50 \%$ in sub-Saharan Africa. Here we are reporting a rare case of primary Tenofovir resistance in a patient with $\mathrm{HBV}$ hepatitis without HIV co-infection.

Methods Patients were 59 years old female, HBV DNA level $5,49,1000 \mathrm{IU} / \mathrm{ml}$ and transaminase high, $\mathrm{kPa}$ scores 8.6, Alpha-fetoprotein level $3.79 \mathrm{IU} / \mathrm{ml}$ and HBeAg level 111.64 .
The patient was started on Tenofovir disoproxil fumarate 300 mg OD. One month HBV DNA level $3210 \mathrm{IU} / \mathrm{ml}, 6$ months HBV DNA level was $674000 \mathrm{IU} / \mathrm{ml}$. The patient was on regular follow up, regularly purchased medicine from the hospital pharmacy, took medicine regularly every single day, no history of other medicine intakes. Clinical evaluation and laboratory findings excluded the presence of other systemic diseases, there was no past history of exposure to Tenofovir. HIV and HCV were negative.

Results Tablet Tenofovir stopped, Tablet Entecavir $1 \mathrm{mg}$ started. Report of mutation study and genotyping revealed A181T/V mutation with A194T and M204V/I, these mutations are associated with resistance to Lamivudine, Adefovir, Tenofovir and there was no reported resistance to Entecavir and Telbivudine. After one month, 3 months and 6 months treatment with Entecavir $1 \mathrm{mg}$ daily, HBV DNA level decreased to $3600 \mathrm{IU} / \mathrm{ml}$, transaminase level normalized on follow up. Possible our patient acquired drug-resistant Hepatitis B virus from patient with HIV-HBV co-infection, the patient taking antiretroviral, or healthcare worker.

Conclusions This is a First report from India of occurrence of Tenofovir mutation A181T/V, A194T and M204V/I in a non HIV infected patient with HBV hepatitis.

\section{IDDF2019-ABS-0208 EXCITING RESULTS WITH INJECTION DARBEPOIETIN ALFA AND PEGFILGRASTIM IN PATIENTS WITH DECOMPENSATED LIVER CIRRHOSIS}

${ }^{1}$ Richa Sharma*, ${ }^{2}$ Vijay Sharma. ${ }^{1}$ Public health foundation of India, Jaipur, Rajasthan, India; ${ }^{2}$ Regional Institute of Health Medicine and Research, Jaipur, Rajasthan, India

\subsection{6/gutjnl-2019-IDDFabstracts.283}

Background Dysregulated erythropoietin (EPO) plasma levels may play a role in the pathophysiology of liver cirrhosis. No report of Darbepoetin alpha and Pegylated Filgrastim use in Liver Cirrhosis. 
Methods To study the benefits of Darbepoetin and Pegfilgrastim in patients with de-compensated liver cirrhosis. Prospectively clinical data recorded since October 2014. Patients with active bleed, hepatorenal syndrome, hepatoma, portal vessel thrombosis were excluded. Patients started on Injection Darbepoetin alpha 200 microgram and Injection Pegfilgrastim $6 \mathrm{mg}$ subcutaneously every 15 days, total of three visits of patients, then three months follow up planned. Improvement in clinical, laboratory parameters analyzed. Median calculated, Wilcoxon Signed-Rank Test applied to compare both groups.

Results $\mathrm{N}=22$ all male, 3 lost to follow up, aetiology of cirrhosis were Non-alcoholic Fatty Liver Disease 5, Hepatitis B Virus 3, Hepatitis C Virus 2 and Alcohol 12. Median age 59years (range: 40 to 70 ). Improvement in Haemoglobin from 10.1 gram $\%$ (range $5.9-13.4 \mathrm{gm} \%$ ) to $10.6 \mathrm{gm} \%$ (range: $7.5-13.7$ gm\%) p value 0.00374 , Total leukocyte count from $5100 / \mathrm{cu}$ $\mathrm{mm}$ to $7100 / \mathrm{cu} \mathrm{mm} \mathrm{p}$ value 0.00214 , Platelet count $90,000 / \mathrm{cu}$ $\mathrm{mm}$ to $146,000 / \mathrm{cu} \mathrm{mm}, \mathrm{p}$ value 0.00096 , INR 1.7 (range 1.54.8 ) to $1.4 \mathrm{p}$ value 0.00064 , Albumin $2.4 \mathrm{gram} / \mathrm{dl}$ (range 1.62.9 ) to $2.5 \mathrm{gm} / \mathrm{dl}$ (range $1.8-3.5$ ) $\mathrm{P}$ value 0.043 , Child score from 10 to $8 \mathrm{P}$ value 0.007 , ascites score 2 to $1, \mathrm{P}$ value 0.00222 . No significant improvement in Serum creatinine, sodium, potassium, calcium, bilirubin, total protein and hepatic encephalopathy. High cost of medicine was the limiting factor. Conclusions Our study suggests that Darbepoetin Alpha and Pegfilgrastim is significantly effective in improving hematology, International normalized ratio, Albumin, ascites and Child score of liver cirrhosis patients.

\section{IDDF2019-ABS-0210 PRELIMINARY EFFICACY AND SAFETY OF 8-WEEK GLECAPREVIR/PIBRENTASVIR IN PATIENTS WITH HCV GENOTYPE 1-6 INFECTION AND COMPENSATED CIRRHOSIS: THE EXPEDITION-8 STUDY}

\begin{abstract}
${ }^{1}$ Robert S Brown Jr*, ${ }^{2}$ Christopher Hezode, ${ }^{3}$ Stanley Wang, ${ }^{4}$ Maria Buti, ${ }^{5}$ WanLong Chuang, ${ }^{6}$ Humberto Aguilar, ${ }^{7}$ Gabor Horvath, ${ }^{8}$ Barbara Rosado Carrion, ${ }^{9}$ Federico Rodriguez-Perez, ${ }^{3}$ Eric Cohen, ${ }^{3}$ Yiran B Hu, ${ }^{3}$ Gretja Schnell, ${ }^{3}$ Chih-Wei Lin, ${ }^{3}$ Lino Rodrigues, ${ }^{3}$ Roger Trinh, ${ }^{3}$ Federico J Mensa. ${ }^{1}$ Weill Cornell Medical College, Center for Liver Disease and Transplantation, USA; ${ }^{2}$ Department of Hepatology, Hôpital Henri Mondor, Université Paris-Est, Paris, France; ${ }^{3}$ AbbVie Inc., North Chicago, Illinois, USA; ${ }^{4}$ Vall d'Hebron University Hospital and CiBERHED del Instituto Carlos III, Barcelona, Spain; ${ }^{5}$ Kaohsiung Medical University Hospital, Kaohsiung Medical University, Kaohsiung, Taiwan; ${ }^{6}$ Louisiana Research Center, Shreveport, LA, USA; ${ }^{7}$ Hepatology Center of Buda, Budapest, Hungary; ${ }^{8}$ Director of GHGCPR Research Institute, Puerto Rico; ${ }^{9}$ GHWC and Gastroenterology Section, San Juan, Puerto Rico
\end{abstract}

10.1136/gutjnl-2019-IDDFabstracts.284

Background The pangenotypic direct-acting antivirals glecaprevir (identified by AbbVie and Enanta) coformulated with pibrentasvir $(\mathrm{G} / \mathrm{P})$ are approved to treat chronic HCV genotype (GT) 1-6 infection. Eight-week G/P achieved high SVR12 rates in Phase 2 and 3 studies, but was not studied in patients with compensated cirrhosis. Based on the high SVR12 rates demonstrated in treatment-naive patients with HCV GT1-6 infection and compensated cirrhosis treated with 12-week G/P, this study evaluates the efficacy and safety of an 8 -week $\mathrm{G} / \mathrm{P}$ treatment duration in that population. Methods EXPEDITION-8 is an ongoing phase 3, non-randomized, single arm, open-label, multicenter study conducted in adults with chronic HCV GT1-6 infection with compensated cirrhosis who are HCV treatment-naive. A recently approved protocol amendment enabled the inclusion of HCV GT3infected patients, who are not included in this analysis. G/P (300 mg/120 mg) is being dosed orally once-daily with food for 8 weeks. The primary efficacy endpoint is the SVR12 rate. Secondary endpoints are the on-treatment virologic failure and relapse rates. Adverse events and clinical laboratory abnormalities are being monitored in all patients.

Results In total, 280 treatment-naive patients with compensated cirrhosis have enrolled and are included in the analysis. To date, 116 patients have completed the post-treatment week 12 visit; (figure 1) shows preliminary efficacy results for those with available post-treatment week 4 and/or 12 data. No virologic failures have occurred. Adverse events (AEs) have been mostly mild, with the most common (at least 5\%) AEs being pruritus and fatigue (both 9\%), headache $(7 \%)$ and nausea (6\%). No AEs have led to discontinuation of G/P; 5 serious AEs have occurred, none of which were deemed related to $\mathrm{G} / \mathrm{P}$.

Conclusions In this ongoing study, G/P for 8 weeks in treatment-naive patients with $\mathrm{HCV}$ infection and compensated cirrhosis has been well-tolerated and achieved high rates of SVR, with no virologic failures to date. Updated efficacy and safety data will be presented at the meeting.

\section{IDDF2019-ABS-0211 EFFICACY AND SAFETY OF GLECAPREVIR/ PIBRENTASVIR IN PATIENTS WITH HCV GENOTYPE 5 OR 6 INFECTION: AN INTEGRATED ANALYSIS OF PHASE 2 AND 3 STUDIES}

${ }^{1}$ Betty B Yao*, ${ }^{2}$ Tarik Asselah, 'Linda Frederick, ${ }^{1}$ Gretja Schnell, ${ }^{3}$ Kris Kowdley, ${ }^{4}$ Paul Y Kwo, ${ }^{5}$ Fred Poordad, ${ }^{6}$ Kinh Nguyen, ${ }^{7}$ Samuel S Lee. ${ }^{1}$ AbbVie Inc., North Chicago, Illinois, USA; ${ }^{2}$ Centre de Recherche sur I'Inflammation, INSERM UMR 1149, Universite Paris Diderot, Department of Hepatology, AP-HP Hôpital Beaujon, Clichy, France; ${ }^{3}$ Swedish Medical Center, Seattle, USA; ${ }^{4}$ Stanford University School of Medicine, Palo Alto, CA, USA; ${ }^{5}$ The Texas Liver Institute, University of Texas Health, San Antonio, TX, USA; ${ }^{6}$ National Hospital for Tropical Diseases, Vietnam; ' University of Calgary, Calgary, AB, Canada

\subsection{6/gutjnl-2019-IDDFabstracts.285}

Background The pangenotypic direct-acting antivirals (DAAs) glecaprevir (identified by AbbVie and Enanta) coformulated

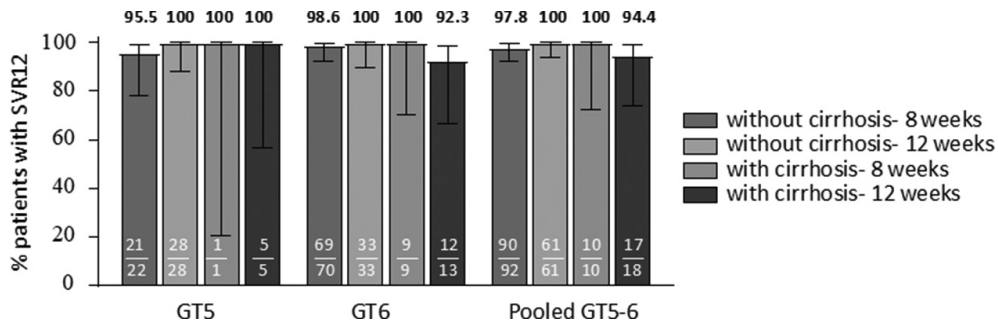

Abstract IDDF2019-ABS-0211 Figure 1 Efficacy with G/P in GT5/GT6 patients 